\title{
CHARACTERISTIC IMPEDANCE AND LOSS DATA FOR A COMMON STRIPI INE PICKUP GEOMETRY *
}

\author{
W. BARRY \\ Continuous Electron Beam Accelerator Facility, 12000 Jefferson Avenue, Newport News, VA 23606, USA
}

S.Y.R. LIU

Virginia Polytechnical Institute and State University, Blacksburg, VA 24061, USA

Received 12 September 1989

Most particle accelerators routinely employ some type of stripline pickups and/or kickers. In this paper, characteristic impedance and resistance per unit length are calculated for a strip transmission line geometry consisting of a circular outer conductor and an infinitely thin concentric inner conductor which subtends an angle $\phi_{0}$ in azimuth (see fig. 1). Useful design plots for these parameters as functions of $\phi_{0}$ and inner to outer conductor radius ratio, $b / a$, are given.

\section{Introduction}

In many particle accelerators, pickups and kickers such as those found in beam position monitors, beam current monitors and stochastic cooling systems ernploy stripline electrodes which interact electromagnetically with the particle beam. The effectiveness of all stripline pickups and kickers, characterized by their shunt impedance, is directly related to the characteristic impedance, $Z_{c}$, of the stripline [1]. In the case of resonant stripline devices where a high $Q$ is desirable, the resistance per unit length, $R^{\prime}$, of the stripline is also an important factor in determining the shunt impedance.

In this paper, $Z_{\mathrm{c}}$ and $R^{\prime}$ are calculated for the stripline electrode geometry shown in fig. 1 . The outer conductor of the strip transmission line consists of a circular beampipe with inner radius $a$. The inner conductor consists of an infinitely thin strip concentric with the beampipe. The inner corductor has radius of curvature $b$ and subtends an angle $\phi_{0}$ about the center of the beampipe. Plots of $Z_{\mathrm{c}}$ and $R^{\prime}$ vs $b / a$ and $\phi_{0}$ for this geometry are presented along with data from approximate formulas for large $\phi_{0}$ and $b / a$ approaching unity.

There are, of course, many mathematical techñiquies for solving this problem. Similar problems have been treated using conformal mapping [2] and spectral domain [3] approaches. In addition, the various Moment Method techniques may be applied [4]. Here, the method of Wang [5] which solves a dual series representation of

* This work was supported by the US Department of Energy under contract DE-AC05-84ER 40150 the boundary conditions is $u$ ied because of its relative simplicity and satisfactory accuracy.

\section{Characteristic impedance and resistance per unit length}

It is well known that transmission lines in the TEM mode support unique voltage and current waves whose properties may be determined solely from a solution to Laplace's equation for the two-dimensional cross section of the line [6]. The stripline geometry of fig. 1 is a two-conductor TEM line supporiing voitage and current waves with amplitudes $V_{0}$ and $I_{0}$ respectively. The current, $I_{0}$, is the total current on either one of the two conductors. The characteristic impedance, $Z_{c}$, is defined to be the ratio $V_{0} / I_{0}$ for the TEM wave. For

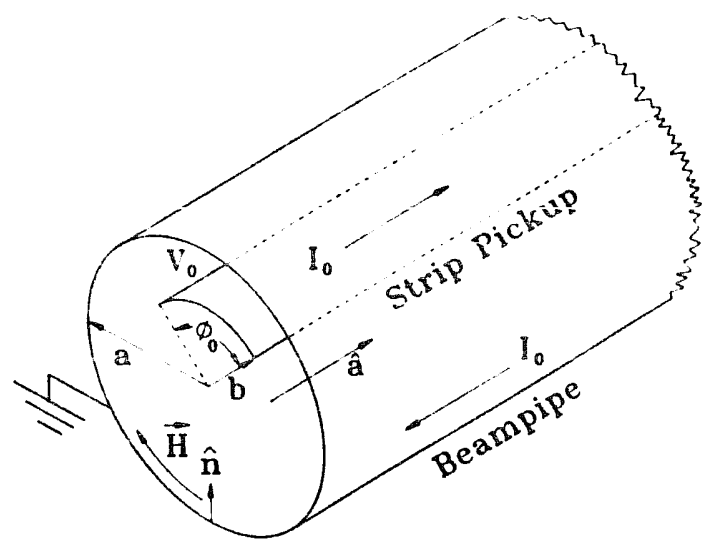

Fig. 1. Schematic of strip transmission line pickup. 


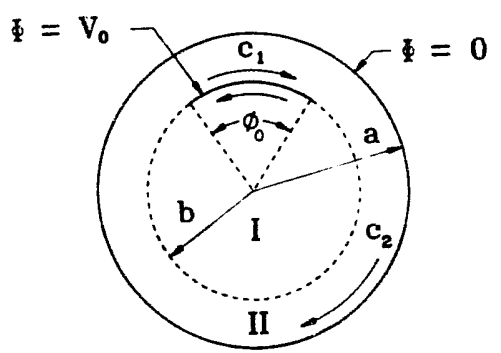

Fig. 2. Geometry for static potential problem.

convenience, the current on the outer conductor is used so that:

$Z_{\mathrm{c}}=\frac{V_{0}}{\oint_{\mathrm{C}_{2}}\left|J_{\mathrm{s}}\right| \mathrm{d} l}$,

where $J_{s}=$ surface current density on $\mathrm{C}_{2}$.

Boundary conditions require that $J_{\mathrm{s}}=\hat{n} \times H$ at $r=$ $a$, where $\hat{n}$ is the unit normal to the outer conductor $\mathrm{C}_{2}$. In addition, for TEM waves, $\hat{a} \times E=\eta \boldsymbol{H}$ where $\hat{\boldsymbol{a}}$ is the unit vector in the direction of propagation and $\eta=120 \pi \Omega$, the wave impedance of free space. Using these relations and the fact that $E$ is everywhere normal to the conducting surface $\mathrm{C}_{2}$, the current may be written:

$I_{0}=\frac{1}{\eta} \oint_{\mathrm{C}_{2}}|\boldsymbol{E}| \mathrm{d} l$.

The electric field may be determined from the solution to the two-dimensionai static potential problem shown in fig. 2. Here, the potential difference beiveen the two conductors corresponds to the amplitude of the TEM voltage wave, $V_{0}$. Using $E=-\nabla \Phi$, the characteristic impedance becomes:

$Z_{\mathrm{c}}=\frac{\eta V_{0}}{\oint_{\mathrm{C}_{2}}|\nabla \Phi| \mathrm{d} l}$.

For the two-conductor oroblem, the solution for $\Phi$ and $-\nabla \Phi$ will always have overall multiplicative factors of $V_{0}$, therefore, $Z_{\mathrm{c}}$ is seen to be a function only of the line geometry.

An approximate expression for the resistance per unit length, $R^{\prime}$, of the stripline may be obtained from power loss considerations. Assuming the beampipe and strip are good enough conductors so that the fields closely resemble the perfectly conducting case, the power lost per unit length of line is:

$\frac{1}{2} R^{\prime} I_{0}^{2}=\frac{R_{\mathrm{s}}}{2} \oint_{\mathrm{C}_{1}+\mathrm{C}_{2}}|H|^{2} \mathrm{~d} l$,

where: $R^{\prime}=$ resistance per unit length, $R_{\mathrm{s}}==$ planer surface resistance, $C_{1}, C_{2}=$ integral paths around each conductor. Using previous arguments and eq. (3), the following expression for $R^{\prime}$ is obtained:

$R^{\prime}=\frac{R_{\mathrm{s}} Z_{\mathrm{c}}^{2}}{\eta^{2} V_{0}^{2}} \oint_{\mathrm{C}_{1}+\mathrm{C}_{2}}|\nabla \Phi|^{2} \mathrm{~d} l$

From eq. (5) it is noted that $R^{\prime}$ is a function only of the stripline geometry and conductor characteristics.

Once again, it is important to point out that expression (5) is an approximation. In particular, two assumptions are made which may result in erroneous calculations of $R^{\prime}$ for certain cases of the stripline geometry. First, the fields used in the calculation are the loss free fields. For the stripline geometry, this results in infinite current densities at the edges of the infinitely thin center conductor. Therefore, contributions to the integral in eq. (5) at the strip edges may be appreciably different from the case for small losses where the fields are high but finite. In addition, expression (5) involves the planer surface resistance, $R_{\mathrm{s}}$. This standard approximation is excellent for the usual cases where the radii of curvature of the surfaces involved are large compared to the skin depth. However, this is not the case at the edges of the strip. Despite the errors resulting from the singular nature of the strip geometry, the $R^{\prime}$ values calculated from eq. (5) are expected to be accurate to within 10\% [7]. Therefore, it is still of some utility to calculate $\boldsymbol{R}^{\prime}$ with the approximate formula in order to gain some insight into the loss behaviour as a function of strip geometry.

It is evident from eqs. (3) and (E) that the parameters of interest are obtained from the solution to the two-dimensional potential problem in fig. 2 . However, before proceeding to solve this problem, it is useful to derive approximate expressions for $Z_{\mathrm{c}}$ and $R^{\prime}$ which are valid for large $\phi_{0}$ and $b / a$ approaching unity. Under these conditions, one might expect the fields to be those of the standard coaxial transmission line only confined to the region $-\phi_{0} / 2 \leq \phi \leq \phi_{0} / 2$ and $b \leq r \leq a$. In this case, neglecting fringing fields, eq. (3) yields:

$Z_{\mathrm{c}} \approx \frac{\eta \ln (a / b)}{\phi_{0}}$.

This equation reduces to the well-known result $Z_{\mathrm{c}}=$ $60 \ln (a / b)$, for $\phi_{0}=2 \pi$. For an approximation to $R^{\prime}$. the same assumptions are made implying that all of the surface current on the inner conductor resides on its outer surface. In this case, eq. (5) gives:

$R^{\prime} \approx \frac{R_{\mathrm{s}}}{a \phi_{0}^{2}}\left(2 \pi+\frac{a}{b} \phi_{0}\right)$

This expression also reduces to the coaxial line case for $\phi_{0}=2 \pi$. 


\section{Solutions for $Z_{\mathrm{c}}$ and $\boldsymbol{R}^{\prime}$}

In order to calculate $Z_{\mathrm{c}}$ and $R^{\prime}$, the potential problem of fig. 2 must be solved. Solutions to Laplace's equation in terms of circular harmonics may be written for the two regions indicated in fig. 2:

$\Phi_{1}(r, \phi)=a_{0}+\sum_{n=1}^{\infty} a_{n} r^{n} \cos n \phi, 0 \leq r \leq b$,

$$
\begin{aligned}
& \Phi_{2}(r, \phi) \\
& =a_{0} \frac{\ln (r / a)}{\ln (b / a)}+\sum_{n=1}^{\infty} a_{n} \frac{\left[r^{n}-\left(a^{2} / r\right)^{n}\right]}{1-(a / b)^{2 n}} \cos n \phi,
\end{aligned}
$$$$
b \leq r \leq a \text {. }
$$

Eqs. (8) and (9) satisfy the boundary conditions $\Phi_{2}(a, \phi)=0, \Phi_{1}(b, \phi)=\Phi_{2}(b, \phi)$ and $\Phi_{1}(0, \phi)$ finite. In addition, even symmetry about $\phi=0$ has been invoked.

In order to determine the unknown coefficients, $a_{n}$, in eqs. (8) and (9), an additional mixed boundary condition must be satisfied at $r=b$ :

$$
\begin{aligned}
& \left.\Phi_{1}\right|_{r=b}=V_{0} \text { on } \quad D_{1} \equiv-\frac{\phi_{0}}{2} \leq \phi \leq \frac{\phi_{0}}{2}, \\
& \left.\frac{\partial \Phi_{1}}{\partial r}\right|_{r=b}=\left.\frac{\partial \Phi_{2}}{\partial r}\right|_{r=b} \text { on } D_{2} \equiv \frac{\phi}{2} \leq \phi \leq 2 \pi-\frac{\phi_{0}}{2} .
\end{aligned}
$$

Eqs. (10) and (11) state that the potential is a constant equal to $V_{0}$ on the inner conductor and the normal component of $E$ is continuous elsewhere on $r=b$. From eqs. (8) and (9):

$$
\begin{aligned}
\frac{\partial \Phi_{1}}{\partial r}= & \sum_{n=1}^{\infty} a_{n} n r^{n-1} \cos n \phi, \\
\frac{\partial \Phi_{2}}{\partial r}= & \frac{a_{0}}{r \ln (b / a)} \\
& +\sum_{n=1}^{\infty} a_{n} n r^{-1} \frac{\left[r^{n}+\left(a^{2} / r\right)^{n}\right]}{1-(a / b)^{2 n}} \cos n \phi .
\end{aligned}
$$

Eqs. (10) and (11) may then be written:

$$
\begin{array}{ll}
X_{0}=1-\sum_{n=1}^{\infty} X_{n} f_{n} & \phi \in D_{1}, \\
X_{0}=2 \ln \frac{b}{a} \sum_{n=1}^{\infty}\left[\frac{X_{n} n}{1-(b / a)^{2 n}}\right] f_{n} & \phi \in D_{2} .
\end{array}
$$

where $X_{n}=a_{n} b^{n} / V_{0}$ and $f_{n}=\cos n \phi$.

To :solve for the unknown coefficients, $X_{n}$, the dual series suations, (14) and (15), are transformed into an infinite system of linear equations by an integration method used by Wang [5]. For notational convenience, the following definitions for weighting functions and inner products are made:

$W_{m}=\cos m \phi$,

$$
\begin{aligned}
& \langle h(\theta), g(\theta)\rangle_{1}=\int_{-\phi_{0} / 2}^{\phi_{0} / 2} h(\theta) g(\theta) \mathrm{d} \theta, \\
& \langle h(\theta), g(\theta)\rangle_{2}=\int_{\phi_{0} / 2}^{2 \pi-\phi_{0} / 2} h(\theta) g(\theta) \mathrm{d} \theta .
\end{aligned}
$$

Multiplying each side of eqs. (14) and (15) by $W_{m}$ and taking the appropriate inner product yields:

$$
\begin{aligned}
& \left\langle W_{m}, X_{0}\right\rangle_{1}=\left\langle W_{m}, 1\right\rangle_{1}-\sum_{n=1}^{\infty} X_{n}\left\langle W_{m}, f_{n}\right\rangle_{1}, \\
& \left\langle W_{m}, X_{0}\right\rangle_{2}=2 \ln \frac{b}{a} \sum_{n=1}^{\infty}\left[\frac{X_{r_{i}} n}{1-(b / a)^{2 n}}\right]\left\langle W_{m}, f_{n}\right\rangle_{2} .
\end{aligned}
$$

Using the properties $\left\langle W_{1,}, X_{0}\right\rangle_{2}=-\left\langle W_{m}, X_{0}\right\rangle_{1}$, $\left\langle W_{m \neq n}, f_{n}\right\rangle_{2}=-\left\langle W_{m \neq n}, f_{n}\right\rangle_{1}$ and $\left\langle W_{m}, f_{m}\right\rangle_{2}=\pi-$ $\left\langle W_{m}, f_{m}\right\rangle_{1}$, eqs. (19) and (20) can be combined to give:

$$
\begin{aligned}
& \sum_{\substack{n=1 \\
n \neq m}}^{\infty} X_{n}\left\langle W_{m}, f_{n}\right\rangle_{1}\left\{1+\frac{2 n \ln (b / a)}{\left[1-(b / a)^{2 n}\right]}\right\} \\
& +X_{m}\left\{\left\langle W_{m}, f_{m}\right\rangle_{1}\right. \\
& \left.-\frac{2 m \ln (b / a)}{\left[1-(b / a)^{2 m !}\right.}\left[\pi-\left\langle W_{m}, f_{m}\right\rangle_{1}\right]\right\} \\
& =\left\langle W_{m}, 1\right\rangle_{1} .
\end{aligned}
$$

Eq. (21) can also be written in matrix form:

$\left[l_{m n}\right]\left[X_{n}\right]=\left[K_{m}\right]$,

where

$$
\begin{aligned}
& K_{m}=\left\langle W_{m}, 1\right\rangle_{1}, \\
& l_{m n}=\left\langle W_{m}, f_{n}\right\rangle_{1}\left\{1+\frac{2 n \ln (b / a)}{\left[1-(b / a)^{2 n}\right]}\right\} \quad m \neq n, \\
& l_{m m}=\left\langle W_{m}, f_{m}\right\rangle_{1}-\frac{2 m \ln (b / a)}{\left[1-(b / a)^{2 n}\right]}\left[\pi-\left\langle W_{m}, f_{m}\right\rangle_{1}\right] .
\end{aligned}
$$

The necessary inner products are easily calculat ed from eq. (17):

$$
\begin{aligned}
& \left\langle w_{m}^{\prime}, 1\right\rangle_{1}=\frac{2}{m} \sin m \phi_{0} / 2, \\
& \left\langle W_{m}, f_{n}\right\rangle_{1}=\frac{\sin (m-n) \phi_{0} / 2}{m-n}+\frac{\sin (m+n) \phi_{0} / 2}{m+n} \\
& m \neq n, \\
& \left\langle W_{m}, f_{n}\right\rangle_{1}=\frac{\phi_{0}}{2}+\frac{1}{2 m} \sin m \phi_{0} .
\end{aligned}
$$

Approximate values for the coefficients $X_{n}$ may now be obtained by truncating eq. (22) to a finite system of equations and finding $[l m n]^{-1}$. 
To find an expression for the characteristic impedance, $Z_{c}$, eq. (13) for $r=a$ is substituted into eq. (3) to give:

$Z_{\mathrm{c}}=\frac{\eta \ln (a / b)}{2 \pi\left|X_{0}\right|}$.

From eq. (29) it is noted that the characteristic impedance of the stripline is that of a standard coaxial line multiplied by the factor $1 /\left|X_{0}\right|$. By comparing eq. (29) with eq. (6), it is seen for large $\phi_{0}$ that $\left|X_{0}\right|$ roughly represents the fraction of $2 \pi$ rad subtended by the stripline center con fuctor. The solution to eq. (22) gives the coefficients, $X_{1}, X_{2}, \ldots, X_{n}$. In order to find an expression for $X_{0}$, eq. (19), which is valid for any $m$, is employed. For convenience, $m=1$ is used, yielding:

$X_{0}=1-\sum_{n=1}^{\infty} X_{n} \frac{\left\langle W_{1}, f_{n}\right\rangle_{1}}{\left\langle W_{1}, 1\right\rangle_{1}}$.

Other values of $m$ work equally well, however, $m=1$ is computationally convenient because $\left\langle W_{1}, 1\right\rangle_{1}$ is nonzero for $0<\phi_{0}<2 \pi$.

The resistance per unit length may be calculated from eq. (5):

$$
\begin{aligned}
R^{\prime}= & \frac{R_{\mathrm{s}} Z_{\mathrm{c}}^{2}}{\eta^{2} V_{0}^{2}}\left\{\int_{-\phi_{0} / 2}^{\phi_{0} / 2}\left|\frac{\partial \Phi_{1}}{\partial r}\right|_{r=b}^{2} b \mathrm{~d} \phi\right. \\
& \left.+\int_{--\phi_{0} / 2}^{\phi_{0} / 2}\left|\frac{\partial \Phi_{z}}{\partial r}\right|_{r=b}^{2} b \mathrm{~d} \phi+\int_{0}^{2}\left|\frac{\partial \Phi_{2}}{\partial r}\right|_{r=a}^{2} a \mathrm{~d} \phi\right\}
\end{aligned}
$$

Using eqs. (12) and (13), an expression for $R^{\prime}$ may be found with some tedious algebra:

$$
\begin{aligned}
R^{\prime}= & \frac{R_{\mathrm{s}} Z_{\mathrm{c}}^{2}}{\eta^{2}}\left\{\frac{1}{b} \sum_{n=1}^{\infty} \sum_{m=1}^{\infty} n m X_{n} X_{m}\left\langle f_{m}, f_{n}\right\rangle_{1}\right. \\
& \times\left[1+\frac{\left(1+(a / b)^{2 n}\right)\left(1+(a / b)^{2 m}\right)}{\left(1-(a / b)^{2 n}\right)\left(1-(a / b)^{2 m}\right)}\right] \\
& +\left(\frac{X_{0}}{\ln (b / a)}\right)^{2}\left(\frac{\phi_{0}}{b}+\frac{2 \pi}{a}\right)+\frac{2 X_{0}}{b \ln (b / a)} \\
& \times \sum_{n=1}^{\infty} n X_{n}\left\langle f_{n}, 1\right\rangle\left[\frac{1+(a / b)^{2 n}}{1-(a / b)^{2 n}}\right] \\
& \left.+\frac{4 \pi}{a} \sum_{n=1}^{\infty} \frac{n^{2} X_{n}^{2}(a / b)^{2 n}}{\left[1-(a / b)^{2 n}\right]^{2}}\right\} .
\end{aligned}
$$

\section{Numerical results for $Z_{\mathrm{c}}$ and $\boldsymbol{R}^{\prime}$}

Numerical calculations for $Z_{c}$ and $R^{\prime}$ were made using 500 terms for the series expansions. The results

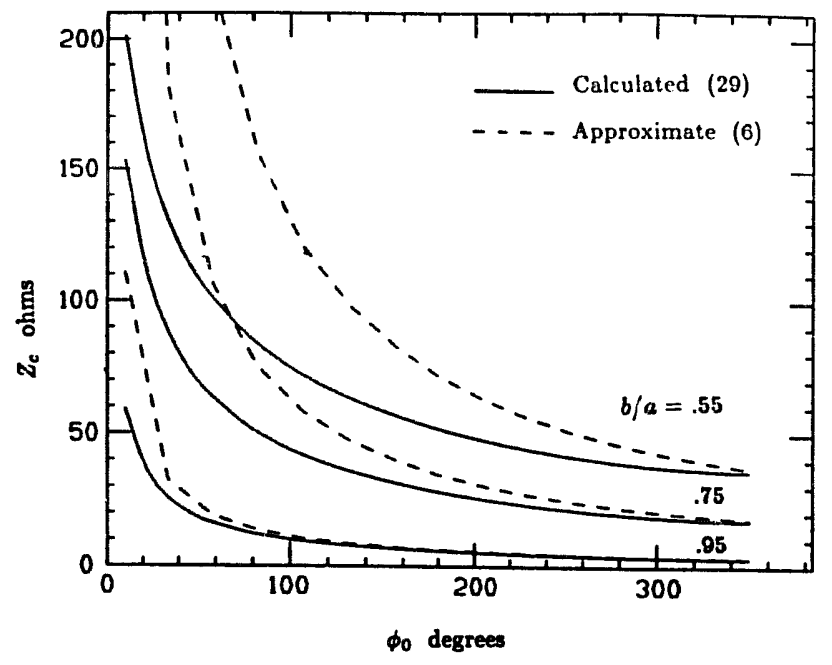

Fig. 3. $Z_{\mathrm{c}}$ vs $\phi_{0}$ for several $b / a$.

for $Z_{\mathrm{c}}$ are shown in figs. 3 and 4 . In fig. $3, Z_{\mathrm{c}}$ is plotted over a wide range of center conductor angles, $\phi_{0}$, for three different $b / a$ ratios. In addition, fig. 3 contains plots of $Z_{\mathrm{c}}$ using the approximate eq. (6). As expected, eq. (6) is seen to be more accurate with increasing $b / a$ and $\phi_{0}$. Fig. 4 shows $Z_{c}$ over a smaller range of $\phi_{0}$ for more $b / a$ ratios.

The accuracy of the data was estimated by studying convergence as the number of coefficients was increased from 100 to 500 in steps of 100 . In all cases the data are believed to be accurate to within 5\% for $\dot{\phi}_{0}>20^{\circ}$. The accuracy also increases dramatically with increasing $\phi_{0}$ because fringe field contributions become more negligable. The worst accuracy is obtained for $b / a>0.8$ and $\phi_{0}<20^{\circ}$. In these cases, the accuracy is estimated at $10-20 \%$. The smaller $b / a$ data is accurate to better than $5 \%$ for all $\phi_{0}$ shown. Lastly, it is pointed out that the calculated values of $Z_{c}$ are always less than the true values.

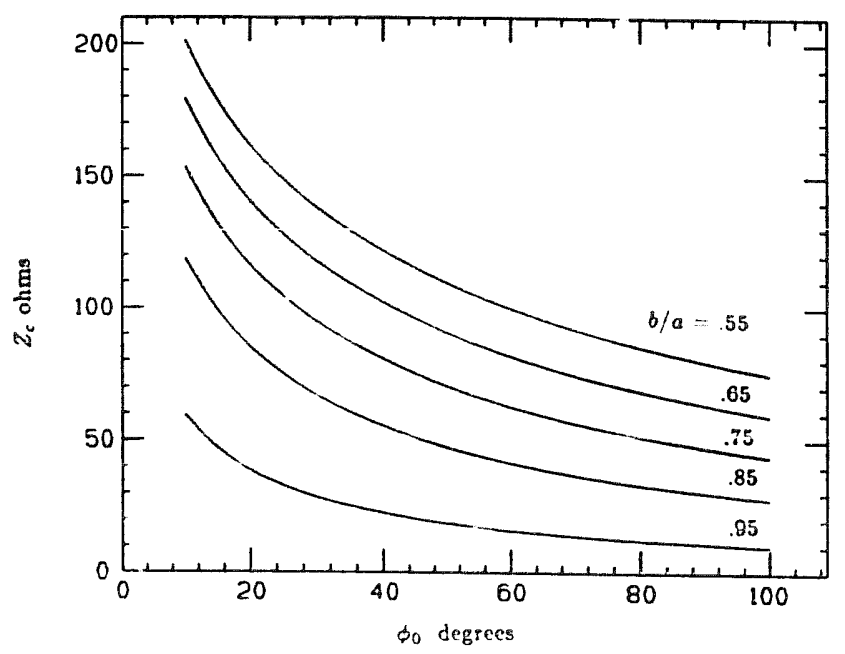

Fig. 4. $Z_{\mathrm{c}}$ vs $\phi_{0}$ for several $b / a$. 


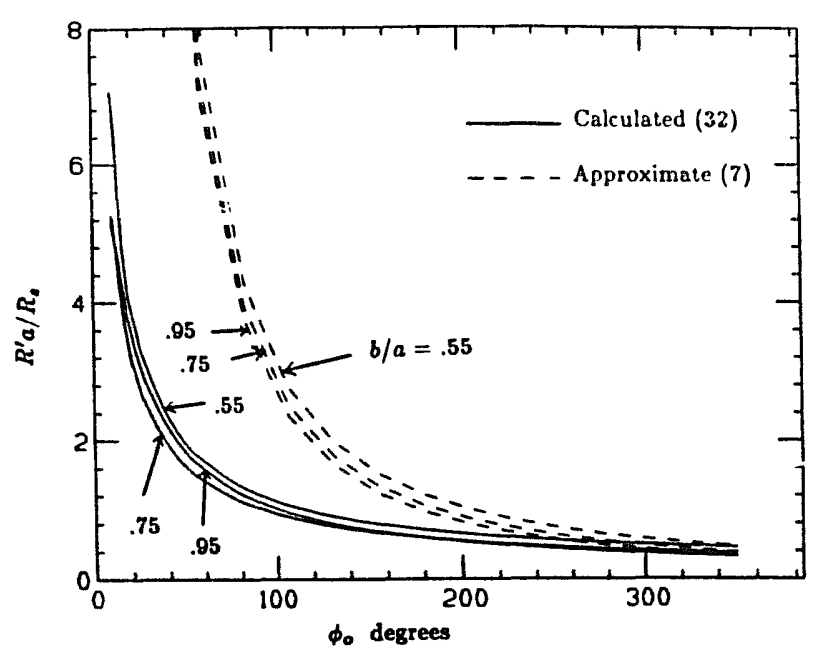

Fig. 5. $R^{\prime} a / R_{\mathrm{s}}$ vs $\phi_{0}$ for several $b / a$.

Because $R^{\prime}$ depends on specific conductor properties and dimensions, the unitless quantity $R^{\prime} a / R_{\mathrm{s}}$ is plotted instead of $R^{\prime}$ in figs. 5 and 6 . In fig. 5 , the approximate values for $R^{\prime} a / R_{\mathrm{s}}$ from eq. (7) are also shown. Again, this approximation is good for large $b / a$ and $\phi_{0}$. In general, the $R^{\prime} a / R_{\mathrm{s}}$ data are not as accurate as the $Z_{\mathrm{c}}$ data because the integrations in eq. (31) involve the edges of the inner conductor. However, it is believed that the data is still good to $5 \%$ except for $b / a>0.8$ and $\phi_{0}<20^{\circ}$. It should be pointed out again that eq. (5) itself is an approximation that may yield $R^{\prime}$ values

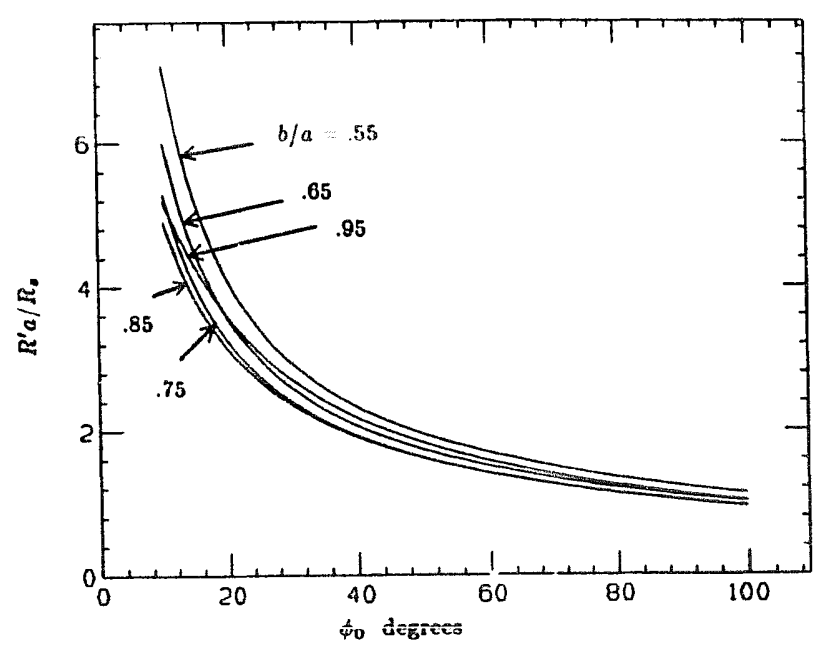

Fig. 6. $R^{\prime} a / R$, vs $\phi \mathbb{r}_{0}$ for several $b / a$. that are to high by $\sim 10 \%$. In view of the fact that the finite number of terms in the calculation yields $R^{\prime} a / R_{\mathrm{s}}$ values that are $5 \%$ lower, it is plausible that the data presented might be only $5-10 \%$ in error from the true resistances.

\section{Discussion}

It should be noted that real pickups and kickers usually contain two or four striplines in one beampipe, one vertical set and/or one horizontal set. However, if $\phi_{0}$ for the strips is reasonably small, say $40^{\circ}$ or less, one might expect the perturbation on the results presented here to be small for the larger $b / a$ ratios. In addition, when calculating shunt impedances for pickups and kickers, one must include the geometric factor, $\mathrm{g}$, which roughly represents the effective fraction of the beam current intercepted by the stripline when operated as a pickup [8]. Using this approximation $\mathrm{g}$ is to first order, equal to $\left|X_{0}\right|$, from eq. (29). Further information on shur.t impedance calculations is contained in ref. [1].

\section{Acknowledgements}

The authors wish to express their gratitude to Sam Heifets, Peter Kloeppel and Geoffrey Kraft of CEBAF for many fruitful discussions.

\section{References}

(1) G.R. Lambertson, Physics of Particle Accelerators, euds. M. Month and M. Dieres, AIP 153, vol. 1 (1987).

[2] D. Homentcoyschi, IEEE Trans. Microwave Theory Techn. MTT-37 (1989) 497.

[3] C.H. Chan and R. Mittra, IEEE Trans. Microwave Theory Techn. MTT-35 (1987) 415.

[4] Harrington, Field Computation by Moment Methods (Robert E. Krieger Publ. Co., Malabar, Florida. 1985).

[5] Yen-Chu Wang, IEEE Trans. Microwave Theory Techn. MTT-26 (1978) 20.

[6] R.E. Collin, Foundations for Microwave Engineering (McGraw-Hill, New York, 1966).

[7] S. Cohn, IRE Trans. Microwave Theory Techn. MTT-3 (1955) IIT.

[8] R.F. Shafer. IFFE. Trans Nucl. Sci. NS-32 (1985) 1933. 